\title{
Analysis of research on satellite network caching mechanism based on ICN
}

\author{
Zhiguo Liu* and Yunqi Li \\ Key Laboratory of Communication Network and Information Processing, Dalian University, Dalian \\ 116622, China
}

Keywords: ICN, Satellite network, Cache mechanism.

\begin{abstract}
ICN was introduced into satellite networks to meet the growing demand for content acquisition. The caching mechanism is one of the most critical parts that profoundly affects the performance of the network. Based on the application background of satellite networks, the working mechanism of ICN and its technical advantages in introducing satellite networks are elaborated. The application status of ICN cache in satellite networks is analyzed to explore the applicability of existing caching mechanism in satellite networks, and the development and challenges of ICN caching mechanism in satellite network are analyzed.
\end{abstract}

\section{Introduction}

With the advancement of technology and the popularity of the Internet, the Internet has become one of the indispensable infrastructures of the information society. Broadband, content and personalization have become the main theme of current network development, and the main needs of the network have gradually changed from information browsing to information sharing. Cisco statistics show that from 2015 to 2020, global mobile data traffic will increase by twice as much as global fixed IP traffic. By 2020 , more than $75 \%$ of the world's mobile data traffic will be video [1].The current TCP/IP network is an end-to-end transmission architecture. This terminal-centric, connection-oriented communication mode gradually does not meet the changes of network requirements. In order to adapt to the network from the sender-driven communication mode to the user-driven content acquisition mode, and better compensate for the problems of the traditional Internet architecture, the researchers propose a new network architecture based on Information Centric Network (ICN). Typical architectures include Data Oriented Network Architecture (DONA) [2], Data-Oriented Translation (DOT) [3], Publish/Subscribe for Internet Routing Paradigm (PSIRP) [4], and Named Data Networking (NDN) [5]. These architectures differ in protocol implementation, but they all follow the same principles. Including: (1) the basic mode is the same: publish-subscribe mode were adopted in the ICN network. (2)Content-based routing: The ICN

\footnotetext{
*Corresponding author: liuzhiguo dldx@163.com
} 
network abandoned the idea of traditional IP networks based on network addresses and designed a routing method based on content names. (3)general intranet cache: Intranet cache refers to the function of the router in the network to cache content resources to the router for request. The entire network is centered on information content, no longer pays attention to the location of the host and directly provides content-oriented services. The goal is to solve the various drawbacks of the traditional network architecture.

With the rapid development of satellite network technology, satellite communication has gradually become an important part of global mobile communications. Satellite communications have been widely used in military, disaster prevention and relief, field exploration and personal mobile communications. Compared with the terrestrial network, the characteristics of the satellite network structure, the topological structure is not fixed, the space span is large, and the switching is frequent, which makes the existing satellite network present various drawbacks. Coupled with the continuous integration of terrestrial networks and satellite networks, satellite networks need to follow the development trend of terrestrial networks and adopt user-driven communication modes. Therefore, the introduction of ICN in satellite networks can not only make up for the shortcomings of existing satellite networks, but also combine with the current evolution trend of terrestrial networks to give full play to the performance of satellite networks. At present, based on the ICN idea, many scholars have carried out many researches and experiments on key technologies such as satellite network architecture, routing and forwarding, and buffering [6,7,8], demonstrating the feasibility of ICN in satellite network from many aspects. Below, we will introduce the ICN working mechanism and the technical advantages in satellite networks in the second section. In the third section, we analyze the current research status of the existing ICN caching mechanism and explore its applicability in the satellite network environment. In the fourth section, we will list the challenges faced by existing satellite network caching mechanisms.

\section{ICN working mechanism and advantage analysis}

The intra-network caching mechanism is one of the most representative features of the ICN network. Intra-network caching can reduce the consumption of network bandwidth, reduce the load on the server, and shorten the time for users to obtain content. Named Data Network (NDN), as one of the more representative future network architectures in the ICN network architecture, has received extensive attention in the academic community. Taking NDN as an example, we will introduces the working mechanism of ICN network caching.

The communication of named data network (NDN) uses content name as a link, and acquires the required content by pulling [9]. Communication is based on two transport packets, the Interest package and the Data package. Under the global naming scheme, content has a globally unique name identifier, each content is segmented in a different block, and each block has an identifier. The identifier includes a content name and a block number. The node completes the information acquisition by matching the content name. The flow of the route cache is described in more detail below with reference to Figure 1: First, assume that none of the content items are cached by the routers in the network. User 1 sends an Interest request to the router R1 connected to it, does not find a match in the local CS of R1, and then find in the PIT table. If there is no match in the PIT, the Interest packet continues to be forwarded to the next hop. Until the content item is matched. The matching Data packet is returned along the request path. The content returned needs to be cached at the router node passing along the path. It is assumed that the content is cached in the R2 router according to a certain caching policy. When the user 2 requests the same content again, the matching is obtained directly from router $\mathrm{R} 2$ and the content is returned without retrieving from the source node. 


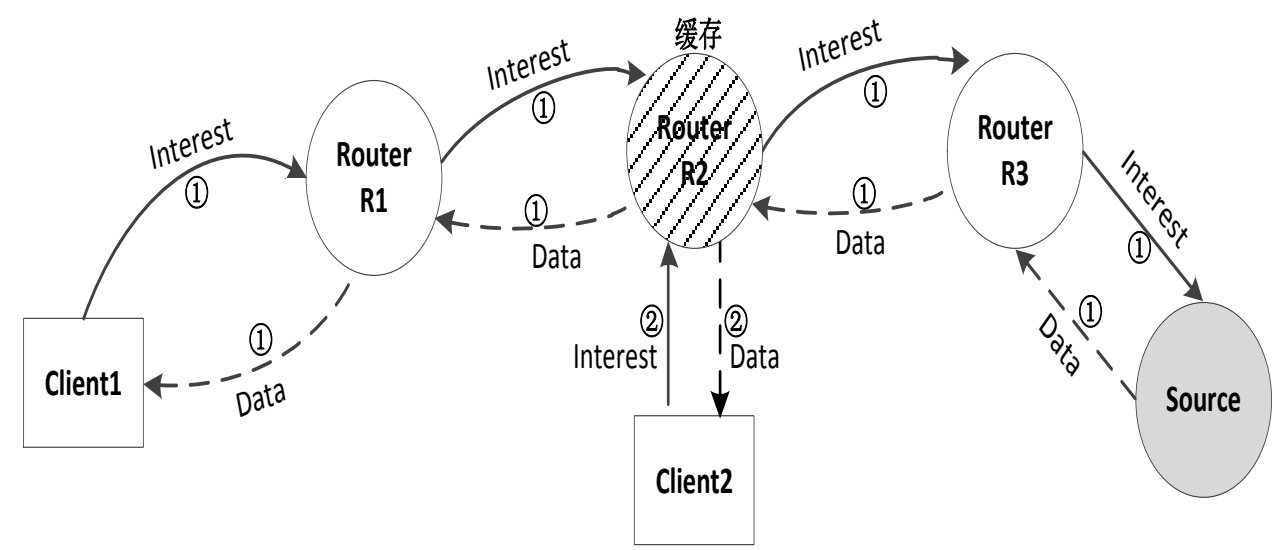

Fig. 1. NDN caching mechanism.

The general ICN working mechanism in terrestrial network is introduced above, and the communication mode of satellite networks is quite different from that of terrestrial networks. The ICN working mechanism cannot be directly applied to the satellite environment. Researchers try to modify the working mechanism of ICN to make it more compatible with the environment of satellite network, so as to bring less communication overhead [10]. And the terrestrial network and the satellite network adopt the unified naming identifier in the network, which can effectively eliminate the communication difference between the ground and the satellite network. Thanks to a unified naming service, satellite networks can implement accurate caching based on name identification. Researchers add a monitoring mechanism on satellite nodes to enable the control side to monitor popular content transmitted by downstream clients and achieve global caching operations. At the same time, ICN adopts data unit-based security protection. Compared with traditional network protection channels and application-based security [11], this mechanism can not only provide better security, but also reduce the additional communication overhead caused by security.

Satellite resources are precious and limited. Reasonable allocation of all kinds of resources on satellite is very important in satellite communication system. The solutions based on ICN are concerned about the importance of caching. The reusability of caching is an important way to improve the performance of content distribution. We know that in traditional connection-based IP networks, the caching of data content requires traversing upstream and downstream data units. We want to be able to cache the required data without the high communication overhead. It is undoubtedly impossible to adopt a traditional network. The network architecture based on the ICN idea can solve this problem well. The satellite experiments built by the researchers confirmed that the use of network caching can lead to better distribution performance $[12,13]$. In this experiment, the use of intra-network caching can reduce traffic in the satellite link. At the same time, the user can obtain data from the cache node in the link, which reduces the repeated transmission caused by the same content. Since ICN supports location-independent unified name identification, content with the same name identification will only be cached once in the entire network. Compared with the traditional network, the satellite network using the intra-network cache can save the satellite storage resources, reduce the communication overhead of the network, and improve the response speed of the content. Table 1 lists the advantages of the ICN working mechanism over traditional IP networks and its advantages in satellite network scenarios. 
Table 1. Advantages of ICN working mechanism and its applicability in satellite networks.

\begin{tabular}{|c|c|c|}
\hline Advantage & Applicability \\
\hline Name & $\begin{array}{c}\text { Name service configuration is } \\
\text { simple. Naming is expansive. }\end{array}$ & $\begin{array}{c}\text { Eliminating Cache Differences between } \\
\text { Satellite and Ground. } \\
\text { Less on-board resource consumption. }\end{array}$ \\
\hline $\begin{array}{c}\text { Routing and } \\
\text { forwarding }\end{array}$ & $\begin{array}{c}\text { Routing is simple and flexible. } \\
\text { More forwarding paths and more } \\
\text { content providers. }\end{array}$ & $\begin{array}{c}\text { The Correspondence of Multipath } \\
\text { Forwarding and Satellite Broadcasting. }\end{array}$ \\
\hline Cache & $\begin{array}{c}\text { Universal Network Cache. } \\
\text { Make full use of cache resources. }\end{array}$ & $\begin{array}{c}\text { Restriction of deployment cache capacity in } \\
\text { conjunction with satellite node equipment. }\end{array}$ \\
\hline Security & $\begin{array}{c}\text { Security Based on Content Name. } \\
\text { No protection on transmission path. }\end{array}$ & $\begin{array}{c}\text { Security Facilities for Reducing Satellites in } \\
\text { Transmission Channels. } \\
\text { Authentication schemes need to be } \\
\text { customized in conjunction with computing } \\
\text { resources. }\end{array}$ \\
\hline
\end{tabular}

\section{Research and analysis of ICN cache application in satellite networks}

\subsection{Cache placement strategy}

In a traditional IP network, the routers in the link can only forward data. The packets are invalidated immediately after they are sent out. The contents of the buffer cannot be reused. The ICN network can cache content to the router for a long time to achieve the reuse of cache resources. In a satellite network environment, this intra-network cache model can make maximum use of the storage resources of the satellite network. The goal of the cache placement strategy is to select which appropriate nodes to cache certain selected content on the path returned by the Data packet to maximize cache revenue. Satellite networks Due to the high dynamics of their nodes and the limited resources, the feasible cache placement strategies in terrestrial networks are generally not applicable to satellite networks. We take the caching strategy in terrestrial network as an example to explore its applicability in satellite network and give us some thoughts.

\subsubsection{Probability-based caching}

The probability-based caching strategy means that on the return path of the Data packet, the cache node is cached at the node with a probability $\mathrm{p}$. The default cache policy of the ICN network is LCE (Leave Copy Everywhere), which requires that the returned content be cached on each node of the return path, resulting in a large number of redundant caches in the entire network [14]. For the problem of high cache redundancy, Eum et al. proposed the RCOne strategy [15], which requires a node to be randomly selected for caching in the content return node. In addition to the fixed probability cache strategy, the researchers try to adjust the factors affecting the cache probability from multiple aspects. For example, a probabilistic cache strategy (MPC) based on content popularity and node-level matching is proposed, considering the factors such as content and node weight [16]. According to the content popularity, the node is assigned a corresponding content proportion. In the popularity-based 
cache, the less popular content tends to be at the very end of the queue, so the low-popular content will be replaced a lot, which will reduce the content diversity of the cache node.

Probability-based cache placement scheme is a tentative method in the early stage of ICN network design. In the complex environment of satellite network, it is difficult to bring better network performance by using only probability-based methods. In the satellite network, it is necessary to combine the current cache state to comprehensively formulate a caching strategy. By increasing the mechanism of listening to the downstream cache state, the researcher enables the upstream satellite nodes to obtain the hot cache content, thus guiding the node to cache [12].

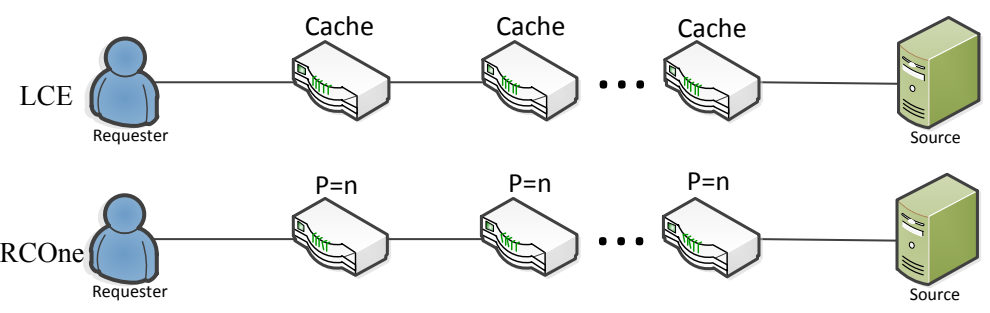

Fig.2. lce and rcone cache strategy schematic.

\subsubsection{Location-based caching}

Location-based caching relies primarily on the location of the cache node in the network to determine whether content is cached at that node. Researchers typically divide the network into two categories: fixed topologies and no fixed topologies. Due to the high dynamics of satellite nodes, a cache placement strategy with a fixed topology is not applicable. Therefore, a scheme without a fixed topology is usually adopted in a satellite network environment. The concept of the edge is used to indicate that the node is closer to the requester (user), thereby proposing to cache the content to the edge of the network so that the content is closest to the user, reducing the response time of the request. Cho et al. proposed a content caching scheme WAVE [17]. The upstream node records the requested number of times in the file, and based on this, suggests what content is cached by the downstream node. Therefore, content with high popularity will be transmitted to edge nodes faster, and the response speed of content will be improved. The LCD policy requires that the cache be cached only on the next hop node of the hit node when the request is acknowledged [18]. Compared to the ICN default policy LCE, cache redundancy can be greatly reduced. In addition, as the request continues, the content is continuously decentralized to the downstream nodes, which keeps the content close to the user. This strategy also improves the speed of the entire network request response while reducing cache redundancy. Still other methods consider the weight of the nodes in the entire network. One of the more representative strategies is the Betw algorithm [19], which measures the importance of a node in the whole network by characterizing the number of nodes through the shortest path number passing through the node.

In a satellite network, the dynamics of the network topology are large, and the cache placement strategy without a fixed topology has certain applicability. The edge node in this kind of caching strategy can be considered as the low-orbit communication satellite closest to the ground. Compared with other orbital altitude satellites, low-orbit satellites have the lowest transmission delay but are more dynamic. However, there is usually no restriction on node dynamics in terrestrial networks. Therefore, location-based caching strategies cannot be directly applied, but they can give some thought to the satellite's hierarchical cache. The 
method of formulating the caching strategy according to the node number is poorly applicable in the satellite network environment. Because of the time-varying of the node median on the same track plane, the relativity of the node median can not bring better network performance. The median of high-orbit nodes is undoubtedly the largest, but due to its number and the limitation of cache resources, it is not feasible to place the cache in high-orbit nodes.

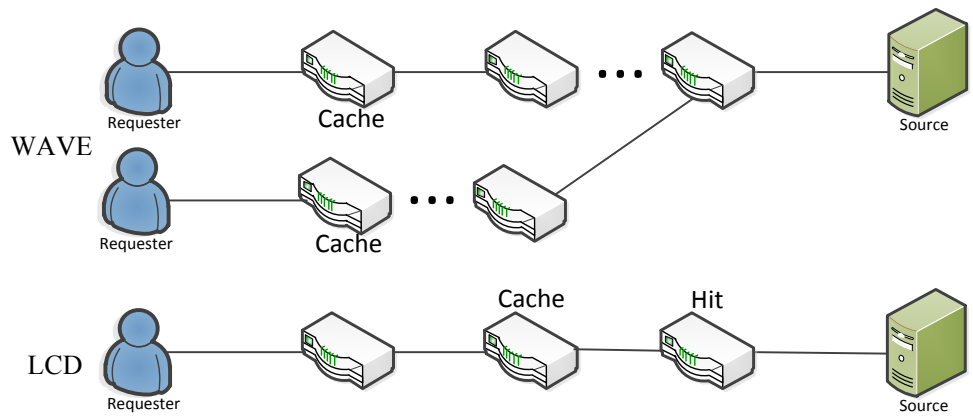

Fig. 3. WAVE and LCD strategy schematics.

\subsubsection{Popularity-based caching}

With the rapid growth of content acquisition applications, the amount of data and users of the Internet have doubled. Coupled with the ICN network architecture that uses intra-network caching, this requires the network to be sensitive enough to changes in content and to be aware of changes in content in a timely and accurate manner. The popularity of content is generally characterized by the number of times a content is requested in a unit of time [20]. The more popular content cached in the network, the higher the hit rate of the network request and the lower the replacement rate of the cache. After ICN is introduced into satellite network, its essence is content-centered, so the research of content popularity is still a hot issue in cache strategy. Therefore, the application of caching strategy in satellite network is better. [21] argues that all users in the network follow the classic Zipf law and that more popular content dominates the network. Simply adopting this model is often not comprehensive enough to describe content popularity.

When we use the content characteristics as the research subject, we need to analyze the characteristics of the content in detail. The popularity of content varies from region to region. For example, regional news or sports may be popular in one region but rarely accessed by users in other regions. The widespread coverage of satellite networks makes this feature more visible in satellite networks. The regional nature of the content should be included as an important factor in the caching strategy of the satellite network. In order to fully utilize the role of cache resources, researchers consider adding a cache state sharing mechanism to save storage resources. This is in line with the very limited storage resource requirements of satellites. In the research of terrestrial networks, it is found that content acquisition is often not from the source server, but from the neighbor nodes of the node to get [22]. The NACID policy establishes a mechanism for obtaining cache information in the neighborhood, and the node can obtain the required data from the nearest (or lowest cost) cache node. The strategy also establishes a repository (Repo), which broadcasts the cache information from the Repo to multiple routers in the neighborhood to achieve the purpose of sharing popularity information in the neighborhood. 
The behavior of the user directly affects the propagation characteristics of the content, so the popularity of the content can also be characterized according to the behavior of the user. The PPC policy uses video user request behavior and leaving behavior as the basis for popularity to measure the relationship between adjacent blocks in the same video file [23]. The popularity is defined as the request behavior and the departure behavior time of a large number of users for a certain content block, and the size of the target content block is used as the mediation of the content block. To calculate the content popularity of each content globally. In addition, there are content-based caching strategies that attempt to classify content to improve cache resource utilization. For example, authors found that some content has low reusability and some content has high timeliness by analyzing the content of the request [24]. Based on this, the content is divided into different categories, and the cache strategy adjusts the proportion of each part of the content in the cache node according to the characteristics of the content class.

In fact, researchers have begun research on content-based popularity in satellite networks very early. The program uses the broadcast nature of satellite communication media, information locality and interest matching table between users and content to develop an information-centric satellite network caching strategy Satcache strategy [25]. Research shows that this strategy can speed up content delivery and reduce the waste of satellite resources. Under the content-centric trend, this strategy is very feasible for satellite networks. Limited by satellite limited network resources and bandwidth, full consideration should be given to the computational resources and bandwidth consumption caused by the strategy.

Table 2. Advantages and disadvantages of mainstream caching strategies and its applicability in satellite networks.

\begin{tabular}{|c|c|c|c|}
\hline Advantage & Insufficient & Applicability \\
\hline Probability-based & $\begin{array}{c}\text { Simple algorithm and } \\
\text { easy implementation }\end{array}$ & $\begin{array}{c}\text { Subjectivity is strong. } \\
\text { Greater uncertainty. }\end{array}$ & $\begin{array}{c}\text { The applicability } \\
\text { is strong, but the } \\
\text { effect is not } \\
\text { obvious. }\end{array}$ \\
\hline Location-based & $\begin{array}{c}\text { Enhanced Web } \\
\text { Experience for } \\
\text { Location Users }\end{array}$ & $\begin{array}{c}\text { Large amount of calculation, } \\
\text { heavy network burden. }\end{array}$ & $\begin{array}{c}\text { The idea of edge } \\
\text { caching has great } \\
\text { applicability. }\end{array}$ \\
\hline Popularity & $\begin{array}{c}\text { Sensitivity to user's } \\
\text { request greatly } \\
\text { reduces the response } \\
\text { delay of popular } \\
\text { content. }\end{array}$ & $\begin{array}{c}\text { Need offline processing, high } \\
\text { computational overhead. }\end{array}$ & $\begin{array}{c}\text { The applicability } \\
\text { is strong, } \\
\text { consumption of } \\
\text { resources should } \\
\text { be considered. }\end{array}$ \\
\hline
\end{tabular}

\subsection{Cache replacement strategy}

Each node in the ICN network has caching capabilities, but its cache space cannot be unlimited. When the node cache space is full, there will be cases where the newly cached content replaces the old content. This limitation exists in both terrestrial and satellite networks, and the background of the problem is the same, so the difference between the two in the cache replacement strategy is small.

LRU is the most commonly used strategy based on access time, which removes the least recently used content. This strategy works well for local areas, but for the entire network, some infrequently accessed content is cached little or not in the network, which seriously affects the request effect of unpopular content. The most representative strategy based on 
access frequency is FIFO, which is "first cache first delete". This strategy is simple, and the replacement order is determined only according to the cache time of the content.

There is also a probability-based replacement strategy that takes into account the poor diversity of content and poor network revenues caused by the above traditional methods. A probability-based caching strategy, such as the random caching strategy Random [26], is used to randomly replace a certain content in the cache, in order to obtain better cache revenue through the strategy. Content-based replacement strategies are often used with content-based placement strategies. When cache replacement occurs, the content with higher popularity is preferentially retained, and the content with low content popularity is replaced. However, the replacement strategy is only implemented according to the popularity of the content. Although the access effect of the popular content is excellent, it will cause the unpopular content to be replaced a lot, resulting in poor content diversity of the entire network. Researchers are also aware of this, so when designing caching strategies, content popularity and other metrics are combined to balance the distribution of popular and unpopular content.

\subsection{Network cache mode}

The ever-increasing number of users and information will inevitably lead to a gradual increase in the cache amount of cache nodes. It is difficult to achieve the best revenue in the entire network for the cache of some central nodes. The network cache mode also needs to be divided into two types: independent cache and collaborative cache according to actual needs.

Stand-alone caching refers to the topology of the entire network as a whole topology. The cache node completely caches a content item in the node, and does not exchange cache status information with other cache nodes. Since the whole network is regarded as a whole, the usage rate of the "important" nodes is naturally higher, and a large number of edge nodes will have a large amount of cache idleness, resulting in waste of cache space. From the perspective of network deployment, the independent caching method is no longer suitable, and will gradually evolve to a collaborative caching method. The collaborative caching method can make full use of the cache resources of the satellite network to improve the performance of the network from another angle.

In the research of terrestrial network, the neighborhood cache placement strategy proposed by authors. When the hot node is cache-replaced, the replaced content is preferentially cached to the neighborhood [27]. In the node, if the capacity of the neighboring node is full, the replaced content is cached to the node with the least heat in the neighborhood. Thereby, the cache cooperation with the neighborhood is completed, and the survival time of the more popular content is guaranteed. In the research of satellite network, the cache placement problem of LEO orbit satellites is combined with the interaction between satellite nodes, and the node exchange matching algorithm is designed to further improve the utilization of satellite node cache resources [28]. The caching method needs to be formulated in combination with the actual situation. Collaborative caching is an effective way to improve cache utilization.

\section{Development and challenges of ICN cache in satellite network environment}

In recent years, ICN-based networks have been proven to bring good results in many research and applications, and the introduction of ICN ideas into satellite networks has also undergone tremendous development. However, there are still many challenges in terms of satellite networks. As the most critical part of the ICN network, network caching plays an important 
role. The following will explore the challenges of caching in conjunction with the development of ICN networks in the context of satellite networks.

\subsection{Mobility restrictions on the caching mechanism}

For the satellite network environment, mobility is still the main problem. This includes the mobility of the satellite nodes and the mobility of the requester. For the mobility of satellite nodes, researchers often offset them through architectural customization. A solution to satellite node mobility is given in [29]. For the mobility of the requester, researchers generally try to use the method of combining with the ground network to design the architecture. In [30][31], the method of cooperation with the ground network facilities is adopted to solve the service of the requester's high-speed mobile. Persistence issues. In [32], the researchers considered a buffer overflow due to satellite mobility and adopted a multi-layer satellite network structure that competes in a non-cooperative manner to improve cache utilization. Existing research is to address the impact of mobility on the network from an architectural perspective. However, the core of ICN is the in-network cache. The cache matching problem caused by mobility will greatly reduce the effectiveness of the caching strategy. Therefore, in the satellite network environment, how to solve the impact of mobility on cached content placement is worthy of further study.

\subsection{Joint cache of satellite and ground}

Satellite networks are rarely used as a stand-alone system. They have long been used in conjunction with terrestrial networks and are often used in areas where terrestrial communications facilities are not available. Software-defined ideas were introduced into the satellite terrestrial network (STN) to coordinate network, cache, and computing resources [33]. After introducing the ICN idea into the satellite network, how to jointly play the role of satellite and terrestrial network caching, and coordinate the cache resources of both to achieve better cache revenue is a very important issue.

\subsection{Cache Security Challenge}

Although ICN can provide content-based secure authentication, there is no need to protect content on the transport channel. However, limited to the computing resources of the satellite nodes, the authentication signature of the content will bring a huge amount of computation, resulting in overload of the router. On the other hand, cache pollution is also a huge challenge for cache security, which caused by two types of attacks. One type is that a cache node is controlled, and after receiving the Interest, the route returns the polluted content, and the content is cached by more cache nodes, thereby causing confusion of the entire network cache. The other is that the attacker predicts future popular content according to the naming rules, manipulates the cache node to issue a large number of requests for the content, and manipulates another cache node to return the polluted content. In turn, the polluted content is cached in other nodes of the network, causing confusion in the cache. The use of satellite nodes is inseparable from the ground network nodes, so the problem caused by cache security is very important. Further research is needed on the security authentication of caches.

\section{Conclusion}

This paper provides research and analysis of ICN caching mechanism in satellite networks. 
The article first introduces the development and background of ICN, and then introduces the working mechanism of ICN cache and its applicability analysis in the satellite network environment. In addition, the existing research on ICN buffer is summarized and classified, and the applicability of ICN in the field of satellite is explored. Finally, the research of the ICN caching mechanism on the satellite network presents the challenges. In summary, the application of ICN in satellite networks is an area worthy of more thinking and research, and the ICN caching mechanism is a crucial part of the architecture. At present, there are many researches on the ICN caching mechanism, but its application in the satellite field is still in its preliminary stage, and theoretical research and experimental verification are not enough. Through the research and analysis of the ICN caching mechanism in the satellite network, the relevant research directions and key issues are clarified, which provides useful ideas and references for the follow-up research.

This research is supported by National Nature Science Foundation of China (61405180402).

\section{References}

1. Cisco, Cisco Visual Networking Index: Forecast and Methodology,(2015-2020) whitepaper, (2016)

2. T. Koponen, M. Chawla, A data-oriented network architecture, in:2007 conference on Applications, technologies, architectures, and protocols for computer communications, Kyoto, (2007),pp.181-192.

3. A. Poutsma, Data-oriented translation, in:18th conference on Computational linguistics, Saarbrücken, (2000) ,pp.635-641

4. D. Lagutin, K. Visala, Publish/Subscribe for Internet: PSIRP perspective, Future Internet Assembly, (2010), pp.75-84

5. L. Zhang, D. Estrin, J Burke, Named data networking (ndn) project, Xerox Palo Alto Research Center-PARC, (2010)

6. Y. Zhang, Y. Wang, SDN Based ICN Architecture for the Future Integration Network, in :2016 16th International Symposium on Communications and Information Technologies (ISCIT), Qingdao, (2016)

7. T. d. Cola, G. Gonzalez, Applicability of ICN-based Network Architectures to Satellite-assisted Emergency Communications, in: 2016 IEEE Global Communications Conference (GLOBECOM), Washington DC, (2016)

8. H. Wu, J. Li , H.C. Lu, A Two-Layer Caching Model for Content Delivery Services in Satellite-Terrestrial Networks, in:2016 IEEE Global Communications Conference (GLOBECOM), Washington DC, (2016)

9. S. Paul, J. Pan, Architectures for the future networks and the next generation Internet: A survey, Computer Communications.34(2011) 2-42

10. A. Detti ,A. Caponi, Exploitation of Information Centric Networking Principles in Satellite Networks, in:2012 IEEE First AESS European Conference on Satellite Telecommunications (ESTEL), Rome, (2012)

11. D. Smetters, V. Jacobson, Securing Network Content, PARC Tech Report, 2009

12. C. N. Ververidis, P.A. Frangoudis, Experimenting with Services over an Information-Centric Integrated Satellite-Terrestrial Network, in: 2013 Future Network \& Mobile Summit, Lisboa, (2013) 
13. V. Priya, B. Sakthisaravanan, Information centric network for secure data transmission in DTN, in: International Confernce on Innovation Information in Computing Technologies, Chennai, (2015)

14. N. Laoutaris ,S. Syntila, Meta algorithms for hierarchical web aches, in: IEEE International Conference on Performance Computing, and Communications, Phoenix, (2004), pp.445-452

15. S Eum, K. Nakauchi, CATT: potential based routing with content caching for ICN, in: second edition of the ICN workshop on Information-centric networking Helsinki, (2012) ,pp.49-54.

16. Y. Li , T. Zhang, Content popularity and node level matched based probability caching for content centric networks, in :2016 IEEE/CIC International Conference on Communications in China (ICCC), Chengdu, (2016)

17. K. Cho, M. Lee, WAVE: Popularity-based and collaborative in-network caching for content-oriented networks, in :2012 Proceedings IEEE INFOCOM Workshops, Orlando, (2012), pp.316-321

18. D. Rossi, G. Rossini, Caching performance of content centric networks under multi-path routing (and more), Telecom ParisTech, (2011)

19. W. K. Chai, D. He, Cache "Less for More" in Information-Centric Networks,in:International Conference on Research in Networking, Berlin Heidelberg,(2012),pp.27-40.

20. T. Wei, L. Chang, MPCS: A mobility/popularity-based caching strategy for information-centric networks, in :2014 IEEE Global Communications Conference, Austin, (2014), pp.4629-4634

21. Y. Kito ,S. Kubota, First challenge of PTP time synchronization experiment through the experimental satellite for communication, in: 2012 International Symposium on Antennas and Propagation (ISAP), Nagoys, (2012), pp.1493-1496

22. A. Pal ,K. Kant, NACID: A Neighborhood Aware Caching and Interest Dissemination in Content Centric Networks, in: 2017 26th International Conference on Computer Communication and Networks (ICCCN), Vancouver, (2017)

23. Y. Zhang, X. Tan, PPC: Popularity Prediction Caching in ICN, in: IEEE Communications Letters, 22(2017)5-8,

24. R. Huo, R. Xie, What to cache: differentiated caching resource allocation and management in information-centric networking, in :China Communications, 13(2016) 261-276

25. L. Galluccio, G. Morabito, Caching in information-centric satellite networks, in: 2012 IEEE International Conference on Communications (ICC), Ottawa, (2012)

26. A. Tatar, Predicting the popularity of online articles based on user comments, in :International Conference on Web Intelligence, Mining and Semantics, Sogndal, (2011)

27. J. Zhi ,J. Li, GAC: Gain-Aware 2-Round Cooperative Caching Approach in Information-Centric Networking, in: 2018 IEEE 37th International Performance Computing and Communications Conference (IPCCC), Orlando, (2018)

28. S. Liu, Distributed Caching Based on Matching Game in LEO Satellite Constellation Networks, in: IEEE Communications Letters,22(2018)300-303 
29. D. Li , H. Li, Virtual Agent Clustering based Mobility Management over the Satellite Networks, in: 2018 10th International Conference on Wireless Communications and Signal Processing (WCSP), Hangzhou, (2018)

30. T. Cola , A. Blanco, ICN-Based Protocol Architectures for Next-Generation Backhauling over Satellite, in :2017 IEEE International Conference on Communications (ICC), Paris, (2017)

31. C. Liang, F. Yu, Information-centric network function virtualization over $5 \mathrm{~g}$ mobile wireless networks,in: IEEE Network,29(2015) 68-74.

32. E. Wang, H. Li, Load Balancing Based on Cache Resource Allocation in Satellite Networks, in: IEEE Access, 7(2019) 56864-56879

33. C. Qiu, H. Yao, Deep Q-learning Aided Networking, Caching, and Computing Resources Allocation in Software-Defined Satellite-Terrestrial Networks, in: IEEE Transactions on Vehicular Technology,68(2019) 5871-5883 